\title{
Nanotechnology in Veterinary Practices
}

Dr. Zakia A M Ahmed

Dr. Mahmoud KHalaf

\section{Dr. Hisham Abdelrahman}

Dept. of veterinary hygiene and management

Faculty of veterinary medicine, Cairo University

\section{Introduction :}

Nanoparticles (NPs) are defined as the science and engineering involved in the design, synthesis, characterization and application of materials and devices whose smallest functional organization ranging between $1-100 \mathrm{~nm}$ in at least one dimension It is the study of materials at the nanoscale. Nanomaterials are best referred to as particles. NT refers to the ability to measure, manipulate and organize matter at the nanoscale level. The scale classically refers to matter in the size range of $1-100 \mathrm{~nm}$, but it is often extended to include materials below $1 \mu \mathrm{m}$ in size .It has the potential to revolutionize veterinary medicine, animal health and other areas of animal production. $(\mathbf{2 5}, \mathbf{2 0}, \mathbf{7}, \mathbf{3 9})$. NT is the design, characterization, production and applications of structures, devices and systems by controlling shape and size at nanometer scale (6). NT is the science and technology of small things $(<100 \mathrm{~nm})$ with new changes in their chemical and physical structure, and also higher reactivity and solubility. Nanostructures are outfitted with smart particles to allow their delivery outside certain biologic barriers such as the brain, skin, eye, mucus, blood, cellular, extracellular matrix placenta, and subcellular organelles. (42). NT holds 
promise for animal health, veterinary medicine, and some areas of animal production. Nanomedicines against various pathogens in veterinary medicine could be developed. Especially, natural nanoantimicrobials such as nano-propolis are useful to veterinary medicine in terms of health, performance, and reliable food production. (40). Veterinary technology has generally established itself well in companionanimal and mixed-animal veterinary medical practice . (9).

Key words: Nanotechnology NT, nanoparticles NPs, nano silwer AgNp, A nanotechnology-derived products NTDPs., nanomaterials .

\section{Uses of Nanotechnology in Veterinary Practices:}

Nanotechnology-based devices for water desalinization have been designed to desalt sea water using at least 10 times less energy than state-of-the art reverse osmosis and at least 100 times less energy than distillation. Nanoscience and engineering could significantly affect molecular understanding of nanoscale processes that take place in the environment; the generation and remediation of environmental problems through control of emissions; the development of new "green" technologies that minimize the production of undesirable by-products; and the remediation of existing waste sites and streams. (35). NT holds promise for animal health, veterinary medicine, and other areas of animal production. Nanotechnology has the potential to impact not only the way we live, but also the way we practice veterinary medicine. (38,22,11). Examples of potential applications of nanotechnology in veterinary combined with livestock disease molecular diagnostics and therapeutics to boost the efficiency 
in the diagnosis and delivery systems treatment of animal disease for improved protein supply and animal food products, new tools for molecular and cellular breeding, modification of animal waste, pathogen detection, and many more. Existing research has demonstrated the feasibility of introducing nanoshells and nanotubes into animals to seek and destroy targeted cells .Nanotechnology operates at the same scale as a virus or a disease-infecting particle, and thus holds the potential for very early detection and eradication. $(\mathbf{1 1}, \mathbf{2 8})$. NPs may be points of advancement for the animal production industry, precautions and should also be taken when considering the employment of nanoparticles for assisting reproduction. When Buffalo sperm (Bubalus bubalis) incubated with $100 \mu \mathrm{g} / \mathrm{mL}$ of titanium oxide NPs reduced viability was recorded. At $10 \mu \mathrm{g} / \mathrm{mL}$ titanium oxide was found to prematurely increase sperm capacitation, which is the final required step in sperm maturation for oocyte penetration and fertilization. (29). Nano-materials have gained special attention in water pollution mitigation researches since last decade. Two vital properties make nanoparticles highly lucrative as sorbents. Nanofiltration techniques are now widely used to remove cations, natural organic matter, biological contaminants, organic pollutants, nitrates and arsenic from groundwater and surface water. Nanomembranes are used to treat contaminated water by filtration or separation techniques. Nanosorbents are widely used as separation media in water purification to remove inorganic and organic pollutants from contaminated water. (6). The nano-propolis is more effective than propolis in terms of antibacterial and antifungal activity. (1). Nano-propolis that is a natural nano-material can be useful to veterinary medicine in 
terms of health, performance, and reliable food production. The nanoparticles are more easily absorbed by the body because they have a size smaller (36). NT has had application in several other sectors, and its application in food and feed science is a recent case. Especially, natural nano antimicrobials obtained from different techniques such as nano-propolis are useful to veterinary medicine in terms of health, performance, and reliable food production. Different nanoencapsulation technologies are used to obtain nanopropolis. Nano-propolis are more easily absorbed by the body because they have a size smaller. Nano-propolis is also more effective than propolis in terms of antibacterial and antifungal activity. Nanotechnology has the potential to solve many mysteries related to animal health, production, reproduction, good hygienic practices during rearing and maintaining of food animals. (40).

NPs are able to direct the antimicrobial active to the mammary gland epitheliums' superficial and to the polymorphonuclear neutrophil compartment (i.e. inside the cells), increasing the concentration of povidine iodine in intracellular compartments against the main mastitis pathogens. A better immune response seems to be obtained with biodegradable nanospheres vaccines produced by conventional methods. Gold NPs are proposed to inhibit the growth of Escherichia coli and Salmonella typhi. Gold NPs dispersed on zeolites eliminate Escherichia coli and Salmonella typhi at short times. Antimicrobial and deodorant sprays recommended for pet clothing (composed of nano TiO2 particles and de-ionized water); for cat and/or dog owner's furniture; for kitty litter; and to keep dog cleaning. In 
mice, which showed a significant reduction in tumor volume through single injections of the radioactive gold nanoparticles.(42).The commercial application of nanotechnology-enabled products NTEPS in the animal health sector is in its infancy, but anticipated applications for companion animals will include diagnostics, targeted drug delivery and effective therapy associated with minimal adverse side effect. A nanotechnology-derived products $N T D P s$ for food-producing animals are expected to focus on modifying animal feeds, maintaining herd health, improving fertility, promoting growth and preserving animal identity. (34). Vaccines using nanoparticles as adjuvants have the further potential to induce both antibody and cellular immune response by simultaneously activating the major histocompatibility complex class I and class II pathways or increase the antigen visibility and their lifespan, to meet the host immune system .(12). Non-traditional antibacterial agents are thus grabbing more attentions and offers great opportunities to overcome resistance. Biosynthesized silver nanoparticles (AgNPs) emerge as viable alternative for treatment of bacterial infections. AgNPs are an effective and rapidly acting factor against a wide variety of common fungi, including genera such as Aspergillus (26, 32).

Magnetic nanoparticles (MNPs) have been successfully used to isolate and group stem cells. Quantum dots have been used for molecular imaging and tracing of stem cells, for delivery of gene or drugs into stem cells, nano materials such as carbon nano tubes, fluorescent CNTs and fluorescent MNPs have been used. With the help of nano medicine early detection and prevention, improved diagnosis, proper treatment and follow-up of diseases is possible. (27). 


\section{Kinds of Applied Nanotech in Veterinary Practices}

Synthetic oligodeoxynucleotides and antigens in biodegradable nanospheres can be used as an alternative approach for immunization. (14). Gold and silver nanoparticles coated with antibodies can regulate the process of membrane receptor internalization. The binding and activation of membrane receptors and subsequent protein expression strongly depend on nanoparticle size.(20). NT tools include microfluidics, nanomaterials, bioanalytical nanosensors etc has the potential to solve many problems related to animal health, production, reproduction, prevention and treatment of animal diseases. Nanoemulsions can find application in the delivery of controlled amounts of drugs into the beverage of breeding animals, prevention of bovine tuberculosis, the controlled release of injectable poorly watersoluble drugs and as destroyer of pathogens. $(\mathbf{1 7 , 3 9 )}$.

Feed additives, Nano-encapsulated food additives include minerals, antimicrobials, vitamins and antioxidants. The most common objective of nanoencapsulation is to enhance the uptake and bioavailability of food additives; other benefits include improving taste, consistency, stability and texture (10). The use of antibiotics as growth promoters in animals brings out problems associated with microbial resistance and antibiotic residue. (15). Nanosilver AgNP as feed additive had positive selective impact on the count of bacteria in poultry digestive tract. On supplementation of 20, 40 and $60 \mathrm{ppm}$ feed, nano-silver caused dose dependent reduction in the weight of the lymphatic organs (3). Nanoselenium has the advantages of high absorption rate, high security, high antioxidant capacity, high egg-laying capacity, 
and good growth performance, and the range between nutrition dose and toxic dose of nano-selenium is significantly wider than that of sodium selenite. (8). In broiler chicken, supplementation of $1.20 \mathrm{mg} \mathrm{kgG} 1 \mathrm{Se}$ (NanoSe) showed a wider range between the optimal and toxic dietary levels of Nano-Se with efficient retention in the body compared to sodium selenite. Addition of nano-Se $(60 \mathrm{~nm})$ to the broiler diet showed an elevation in survival rate, average daily gain and feed to gain ratio with $0.15-1.20 \mathrm{mg} \mathrm{kgG} 1 \mathrm{Se}$ concentration37. In layer chicks, nano-Se of $0.3 \mathrm{mg} \mathrm{kgG1}$ of dry diet was found to have better physiological effects (24). A study on nano zinc showed that supplementation of $0.06 \mathrm{ppm}$ in the basal diet of broiler birds showed improved immune status and bioavailability compared to inorganic zinc.(37). When broiler birds are fed with nano form of calcium phosphate by replacing up to $50 \%$ requirement of dicalcium phosphate, they showed a best feed conversion ratio $(1.39 \pm 0.02)$ and differed significantly from the control groups $(\mathrm{p}<0.05) .(44)$.

The application of NT in animal feeding includes the use of different NPs in the administration of medication, nutrients, probiotics, supplements and other substances. Recently, feed additives such as trace minerals in the form of nanoparticles can be effectively used to fulfill the requirement of minerals in the livestock and poultry feed. Nano-additives can also be incorporated in micelles or capsules of protein or another natural feed ingredient. (18).

Nanosensors and nanovaccines, adjuvants, gene delivery and smart drug delivery methods have the potential to revolutionize animal health and production. There can be numerous applications of the technology for disease diagnosis, 
treatment, drug delivery, animal nutrition, animal breeding, reproduction, tissue engineering and value addition to animal products .A nanocomposite of $\mathrm{MgO}-\mathrm{SiO} 2$ has been used as an effective adsorbing agent for removal of aflatoxin from wheat flour. (23). Similarly, a modified montmorillonite nanocomposite has been used to reduce the toxicity due to aflatoxin in feeds of broiler chicks. $(\mathbf{4 1 , 2 3 )}$.

Nanoscale devices may be able to penetrate biological barriers such as the blood-brain barrier or the stomach epithelium, barriers that normally make it difficult for therapeutic and imaging agents to reach certain tumours. (39). Nanoparticle-based drug delivery improves the solubility of poorly water-soluble drugs, prolongs the half-life of drug, releases drugs at a sustained rate or in an environmentally responsive manner and thus lowers the frequency of administration, delivers drugs in a target manner to minimize systemic side effects, and delivers two or more drugs simultaneously for combination therapy to generate a synergistic effect and suppress drug resistance (2). The precise delivery and controlled release of drugs, resulting in a small footprint in animal waste and the environment, which would otherwise result in antibiotic resistance. It reduced environmental concerns associated with the use of antibiotics and enabled new drug administration's that are easy, quick, non-intrusive to animals and most importantly, economical.(13).Conventional adjuvants are not tuneable. The nanoparticle-based adjuvants can be engineered for reduced dosage frequency and a convenient administration route in order to provoke a target specific immune response, e.g. the intranasal route to better target mucosal immunity.(43). The 
NanoPatch ${ }^{\circledR}$ is an example of a nanotechnology-enabled device that delivers vaccines dermally to humans though the concept applies equally to animals. (16).

Manufactured nanomaterials were carbonaceous nanomaterials (eg carbon nanotubes), semiconductors (eg quantum dots), metal oxides (eg zinc oxide), nanopolymers (eg dendrimers), nanoclays, emulsions (eg acrylic latex) and metals (e.g silver).They noted that these nanomaterials may exist in single, aggregated, or agglomerated forms and have various shapes, coatings and surface functionality. (5). Nanosponges' and 'nanojuice' have been reported in recent scientific literature. 'Nanosponges' are approximately 3000 times smaller than a red blood cell and comprise a biocompatible polymer core coated with segments of red blood cell membranes derived from the host. (20).

Nanoshells, the active gold coated glass particles commonly known as nanoshells could help veterinary medicine to improve the treatment of parasites facilitating the target of the parasite and the drug delivery, physically destroying the infection. Nanoshells can be useful in the study of host-parasite as biosensors. Livestock, pets and wildlife may benefit from products in nanoscale, such as vaccines, target recombinant proteins, or new drug candidates. Nanotechnology has given the tools to medicine to improve treatments and to develop effective immune responses against infectious agents. Cancer research has been the major area met by nanotechnology. Diseases caused by bacteria, fungi and viruses have also been targets for nanoparticles. Nanotech can offer an improvement in imaging and diagnosis of the diseases as well, slipping away the limitations of biological barriers. (12). A few types of NPs including polymeric NPs, 
lipid NPs liposomes, dendrimers , carbon materials, and magnetic NPs have been widely investigated as drug delivery platforms, of which several products have been introduced into pharmaceutical market.(21). $A g N P$ has been used in animal breeding as a disinfecting agent used to sanitize transport chambers or the space used for the storage of animals. The nanosilver preparation applied for the disinfection of eggs and hatchers reduced microbiological contamination. The preparation used showed bactericidal and fungicidal effectiveness comparable to UV radiation, and its effectiveness increased throughout the incubation. In the case of fungi, on the 7th day of incubation, fogging with AgNPS gave better protection to the surface of eggs than irradiation with UV. Very good results were achieved in the case of organic gaseous contaminants. After application of the nanosilver preparation, these levels decreased by $86 \%$. The level of contaminants in the air inside the incubator decontaminated with UV was $40 \%$ higher than in the incubator disinfected with AgNPS. The application of AgNPS may be used to optimize the process of incubation. Nanosilver is not able to replace efficiently functioning ventilation but may be an element supporting the elimination of gaseous contaminants. (30). A nanoparticle made from the canola protein cruciferin has been investigated and demonstrated that these nanoparticles could encapsulate both hydrophobic and hydrophilic bioactive compounds, protect them from a simulated stomach environment, and release them in a simulated intestinal environment. (4). 
Metal NPs have drawn the attention of the medical field for their use in imaging and as antimicrobial therapies that lyse Gram positive and Gram negative bacterial cell walls. (31). Non-traditional antibacterial agents are thus grabbing more attentions and offers great opportunities to overcome resistance. Biosynthesized silver nanoparticles (AgNPs) emerge as viable alternative for treatment of bacterial infections. The synthesized silk AgNPS show an effective antibacterial activity against pathogens of Gram positive and Gram negative bacteria. AgNPs undergo an interaction with bacterial cell and displayed the strong action against MRSA and E. coli. The antimicrobial properties of silver compounds and silver ions had been historically recognized and applied in a wide range of applications from disinfecting medical devices and for the treatment of water. AgNPs seem to be alternative antibacterial agents to antibiotics and have the ability to overcome the bacterial resistance against antibiotics. (32). The synthesized silk silver nanoparticles show an effective antibacterial activity against pathogens of Gram positive and Gram negative bacteria. The result suggests that silver nanoparticles undergo an interaction with bacterial cell and displayed the strong action against MRSA and E. coli. (33).

During the last decade, titanium dioxide (TiO2) nanoparticles have emerged as promising photocatalysts for water purification. Consequently, different workers had adopted the methods such as chemical precipitation, sol-gel, vapour deposition, solvo thermal, solid state reaction etc. for the synthesis of some nanostructured mixed oxides, which can be effectively used for groundwater treatment. (6). 


\section{References}

1. Afrouzan. H., Amirinia C., Mirhadi ,S.A., Ebadollahi ,A, Vaseji. N and Tahmasbi G. (2012). Evaluation of antimicrobial activity of propolis and nanopropolis against Staphylococcus aureus and Candida albicans. Afr J Microbiol Res. 6:421-425.

2. Agnieszka, Z.,Wilczewska1.,Niemirowicz, K., Markiewicz K.H and Car.H (2012). Nanoparticles as drug delivery systems. Pharmacological Reports,64: 1020-1037.

3. Ahmadi, F., (2012). Impact of different levels of silver nanoparticles (Ag-NPs) on performance, oxidative enzymes and blood parameters in broiler chicks. Pak. Vet. J., 32: 325-328.

4. Akbari, A and Wu, J. (2016).Cruciferin nanoparticles: preparation, characterization and their potential application in delivery of bioactive compounds. Food Hydrocoll.;54:107-18.

5. Batley.GE, Kirby.JK and McLaughlin.MJ.(2012) Fate and risks of nanomaterials in aquatic and terrestrial environments. Accounts of Chemical Research 46(3): pp 854-62.

6. Bhattacharya1, S, Saha .I.I, Mukhopadhyay.A., ChattopadhyayD., Ghosh.U.C and Chatterjee.D. (2013) .Role of nanotechnology in water treatment and purification: Potential applications and implications. International Journal of Chemical Science and Technology 2013; 3(3): 59-64.

7. Boulaiz. H, Alvarez ,P.J, Ramirez. A, Marchal J.A, Prados J, Rodríguez-Serrano F, Perán M, Melguizo 
C and Antonia Aranega (2011). Nanomedicine: Application Areas and Development Prospects. International Journal of Molecular Sciences, 12: 33033321.

8. Cai. C, Qu X.Y, Wei, Y.H and Yang, A.Q (2013) Nano-selenium: nutritional characteristics and application in chickens. Chin J Anim Nutr 12:28182823.

9. Chadderdon, L.M, Lloyd J.W, Pazak HE.(2014). New directions for veterinary technology. J Vet Med Educ ;41(1):96-101.

10.Chaudhry Q, Scotter M., Blackburn J, et al. (2008) Applications and implications of nanotechnologies for the food sector. Food Additives and Contaminants. Part A, Chemistry, Analysis, Control, Exposure and Risk Assessment 25(3): pp 241-58.

\section{Chakravarthi.V.P and Balaji. S.N.}

(2010).Applications of Nanotechnology in Veterinary Medicine. Veterinary World, 2010, Vol.3(10):477-480.

\section{Cruz ,A.A and Molento, M.B.(2015).}

Nanotechnology: meeting the future of Veterinary Parasitology Research. Pesq. Vet. Bras. vol.35 no.10 Rio de Janeiro.

13.Ditta, A (2012). How helpful is nanotechnology in agriculture? REVIEW. Adv. Nat. Sci.: Nanosci. Nanotechnol. 3 , 033002 (10pp).

14.Diwan, M.; Tafaghodi, M and Samuel, J. (2002). Enhancement of immune responses by co-delivery of A $\mathrm{CpG}$ oligodeoxynucleotide and tetanus toxoid in biodegradable nanospheres. J. Control. Release. v.85, n.1-3; p.247-262. 
15.Fahri, V.R. (2009). Potensi Nanopropolis Trigona Spp. Asal Bukittinggi Sebagai Pemacu Pertumbuhan Pada Tikus Putih (Sprague-Dawley). Bogor (Indonesia): Bogor Agricultural University.

16.Fernando GJP, Chen X, Primero CA, et al. (2012). Nanopatch targeted delivery of both antigen and adjuvant to skin synergistically drives enhanced antibody responses. Journal of Controlled Release 159(2): pp 215-21. doi: 10.1016/j.jconrel.2012.01.030.

17.Ganguly, S. and Mukhopadhayay S.K.(2011). Nano Science and Nanotechnology: Journey from Past to Present and Prospect in Veterinary Science and Medicine . International Journal of NanoScience and Nanotechnology. Volume 2, Number 1, pp. 79-83.

18.Gopi M., Pearlin B., Kumar ED., Shanmathy,M and Prabakar,G.(2017). Role of Nanoparticles in Animal and Poultry Nutrition: Modes of Action and Applications in Formulating Feed Additives and Food Processing. International Journal of Pharmacology, 13: 724-731.

19.Hu C-MJ, Fang RH, Copp J, et al. (2013) A biomimetic nanosponge that absorbs pore-forming toxins. Nature Nanotechnology 8(5): pp 336-40. doi: 10.1038/nnano..54.

20..JIANG.W., KIM1, B.S ., RUTKA3.J.T, and . .W.C.W.( 2008 ).Nanoparticle-mediated cellular response is size-dependent . Nature Nanotechnology 3(3):145-50. 
21.Kumar,P. S (2016). Nano-drug delivery system: applications in veterinary medicine and animal health. International Journal of Science, Environment and Technology, Vol. 5, No 6, , 4447 - 4451.

22.Kuzma J. (2010). Nanotechnology in animal production- Upstream assessment of applications. Livestock Sci. 130:14-24.

23.Manuja A, Kumar B and Singh RK. (2012). Nanotechnology developments: opportunities for animal health and production. Nanotechnol Dev. 2:1725.

24.Mohapatra, P., R.K. Swain, S.K. Mishra, T. Behera and P. Swain et al., (2014). Effects of dietary nanoselenium supplementation on the performance of layer grower birds. Asian J. Anim. Vet. Adv., 9: 641-652.

25.Muller, R.H., Mader, K. and Gohla,S (2000). Solid lipid nanoparticles (SLN) for controlled drug delivery-a review of the state of the art. European journal of pharmaceutics and biopharmaceutics, 50: 161-177.

26. Naghsh N., M. Safari, and P. Hajmehrabi,( 2012). "Comparison of nanosilver inhibitory effects growth between Aspergillus niger and E. coli," Indian Journal of Science and Technology, vol. 5, no. 3, pp. 2448-2450.

27. Nikalje ,A.P (2015) Nanotechnology and its Applications in Medicine. Med chem. 5:081089. 
28.Num, S.M and Useh N.M. (2013). Nanotechnology applications in veterinary diagnostics and therapeutics. Sokoto J Vet Sci. 11:10-14.

29.Pawar K and Kaul G. (2012) .Toxicity of titanium oxide nanoparticles causes functionality and DNA damage in buffalo (Bubalus bubalis) sperm in vitro. Toxicol Ind Health.; doi:10.177/0748233712462475.

30.Pulit-Prociak.J and Banach.M .(2016). Silver nanoparticles - a material of the future...? Open Chem., ; 14: 76-91.

31.Ramasamy M, Kim S, Lee SS and Kee D. (2016). Recyclable photo-thermal nano-aggregates of magnetic nanoparticle conjugated gold nanorods for effective pathogenic bacteria lysis. J Nanosci Nanotechnol.;16:555-61.

32.Rani R, Sharma D, Chaturvedi $M$ and Yadav J.P.(2017).Green Synthesis, Characterization and Antibacterial Activity of Silver Nanoparticles of Endophytic Fungi Aspergillus terreus. J Nanomed Nanotechnol 8: 457.

33.Reddy, H A and Venkatappa B (2017). Synthesis of Silk Silver Nanoparticles form Silkworm Cocoons and Their Antibacterial Activity on Methicillin Resistant Staphylococcus aureus (MRSA) and Escherichia coli. J Nanomed Nanotechnol, 8:4.

34.Reeves, $P$ (2014). Regulatory considerations for nanopesticides and veterinary nanomedicines. A Draft APVMA Report. Australian Pesticides \& Veterinary Medicines Authority. 
35.Roco M.C and Bainbridge W.S. (2001). Societal implications of Nanoscience and nanotechnology. National science foundation. International Technology Research Institute, World Technology (WTEC) Division, Loyola College.

36.Sahlan M, Dienayati D, Hamdi D, Zahra S, Hermansyah $H$ and Chulasiri M. (2017) .Encapsulation process of propolis extract by casein micelle improves sunscreen activity. Makara J Technol. $21: 1-6$.

37.Sahoo, A., R.K. Swain and S.K. Mishra, (2014).. Effect of inorganic, organic and nano zinc supplemented diets on bioavailability and immunity status of broilers. Int. J. Adv. Res., 2: 828-837.

38.Scott, N.R. (2005). Nanotechnology and animal health. Rev Off Int Epizoot. 24:425-432.

39.Sekhon, B.S. (2012). Nanoprobes and Their Applications in Veterinary Medicine and Animal Health. Research Journal of Nanoscience and Nanotechnology, 2: 1-16.

40.Seven.P.T., Seven.I., Baykalir.B.G., Mutlu.S.I and Salem.A.Z.M.(2018). Nanotechnology and nanopropolis in animal production and health: an overview. Italian Journal Of Animal Science, p:1-10. (Print) 1828$051 X$.

41.Shi, Y.H, Xu, Z.R, Feng, J.L and Wng CZ. (2006). Efficacy of modified montmorillonite nanocomposite to reduce the toxicity of aflatoxin in broiler chicks. Anim Feed Sci Technol. 129:138-148.

42.Troncarelli MZ, Brand ao HM, Gern JC, Guimar aes AS and Langoni H. (2013). 
Nanotechnology and antimicrobials in veterinary medicine. In: M_endez-Vilas A, editor. Microbial pathogens and strategies for combating them: science, technology and education. Badajoz: Formatex Research Center; p.543-556.

\section{Underwood $C$ and van Eps AW (2012).} Nanomedicine and veterinary science: The reality and the practicality. Veterinary Journal, 193: 12-23.

44.Vijayakumar, M.P. and Balakrishnan, V. (2014). Effect of calcium phosphate nanoparticles supplementation on growth performance of broiler chicken. Indian J. Sci. Technol., 7: 1149-1154. 